\title{
Verhaltenstherapie
}

Verhaltenstherapie 1998;8:144-147

\section{Arbeitsgemeinschaft für Verhaltensmodifikation Schweiz (AVM-CH)}

\section{Weiterbildungskurse in kognitiver Verhaltenstherapie und Verhaltensmedizin}

Für PsychologInnen und ÄrztInnen (und andere Berufsgruppen aus dem psychosozialen Bereich) bietet die AVM-CH zwei bzw. vierjährige Aus- und Weiterbildungsgänge an. Diese umfassen «kombinierte theoretisch-praktische Kurse», «Supervision» und «Selbsterfahrung». Dabei wird qualifiziertes Lehrpersonal mit der Durchführung der Veranstaltungen beauftragt.

Den Weiterbildungsgängen für PsychologInnen bzw. ÄrztInnen liegt ein Curriculum zugrunde, das sich an den aktuellen Richtlinien der FSP bzw. FMH orientiert.

Kombinierte theoretisch-praktische Kurse für das Wintersemester 1998/99 (für den neuen, dritten Weiterbildungsgang und weitere InteressentInnen)

1. Einführung in die Verhaltenstherapie

3./4. 10.98

(Dipl.-Psych. N. Kienzle, Heckscher Klinik München)

2. Problem- und Verhaltensanalyse/Therapieplanung

28./29. 11.98

(Prof. Dr. phil. Dr. Schulte, Dipl.-Psych., Universität Bochum)

3. (Lern-) Theoretische Grundlagen der Verhaltenstherapie/medizin 12./13. 12.98

(Dipl.-Psych. K. Mayer, Tagesklinik Winterthur)

4. Standardmethoden der Verhaltenstherapie und Indikationsfragen I 6./7.2. 99

- Rollenspiel, Entspannungsverfahren, Problemlösetherapie, etc.

(Dr. phil. S. Fliegel, Dipl.-Psych., Universität Bochum)

Anmeldeunterlagen für den 3. Weiterbildungsgang der AVM-CH und weitere Infos:

Frau F. Perret

AVM-CH Sekretariat

Universitäre Psychiatrische Dienste (UPD)

Bolligenstr. 111

CH-3000 Bern 60

Tel.: 031 / 9309915

Fax: 031 / 9309988

\section{Ausbildungsseminare zum Integrierten Psychologi- schen Therapieprogramm (IPT) für schizophrene Patien- ten an den Universitären Psychiatrischen Diensten (UPD) Bern}

\section{Semianr 1: Kognitive Therapie}

Nach einem Überblick über das therapierelevante Grundlagenwissen zur Schizophrenie vermittelt dieses Seminar die Unterprogramme «Kognitive Differenzierung» und «Soziale Wahrnehmung» des IPT. Das Konzept einer Spezialabteilung, bei der u. a. das IPT zum Einsatz kommt, wird vorgestellt.

Termin: 13.-15. November 98

\section{Seminar 2: Therapie sozialer Kompetenz}

Dieses Seminar behandelt folgende Unterprogramme des IPT: «Verbale Kommunikation», «Soziale Fertigkeiten», «Interpersonelles Problemlösen». Zusätzlich wird die Weiterentwicklung des IPT (Therapiemanuale zur gezielten Wohn-, Arbeits- und Freizeitrehabilitation; Umgang mit Emotionen) vorgestellt.

Termin: 15.-17. Januar 99

Neben den unter beiden Seminaren angegebenen Inhalten werden in jedem Kurs folgende Themen angesprochen: Indikationsstellung der einzelnen Unterprogramme; Verfahren zur therapiebegleitenden Diagnostik und Effizienzkontrolle; empirische Untersuchungen zum IPT; Umgang mit schwierigen Patienten; gruppendynamische Gesichtspunkte; Therapieplanung und Gruppenzusammensetzung; Durchführungsmodalitäten.

Die Teilnehmer lernen die einzelnen Unterprogramme über Demonstrationen mit Rollenspielen und Videobeispiele in Groß- und Kleingruppenarbeit kennen. Therapeutenverhalten wird dabei eingeübt. Es wird erwartet, daß sich die Teilnehmer kritisch-reflektierend mit den Seminarinhalten auseinandersetzen.

Referenten: Volker Roder, Bettina Hodel Kosten: $\quad 390,-$ CHF (pro Seminar)

Hinweis: Beide Seminare können unabhängig voneinander besucht werden. Für die Teilnahme an Seminar 2 sind jedoch Vorkenntnisse zum IPT erforderlich.

Literatur: Roder V, Brenner HD, Kienzle N, Hodel B: Integriertes Psychologisches Therapieprogramm für schizophrene Patienten (IPT). 4. überarb. Aufl., Weinheim, PVU, 1997. 
Weitere Informationen, Anmeldung:

Dr. Volker Roder

Universitäre Psychiatrische Dienste Bern

Direktion Mitte/West

Bolligenstr. 111

CH-3000 Bern 60

Fax: 031 / 93 09-988

\section{Arbeitsgemeinschaft für Verhaltensmodifikation e. V. (AVM-D)}

Die nächste $A K$-Leiter- und Lehrtherapeutenkonferenz findet am 27. 6. 98 in Nürnberg statt.

Die Einladungen dazu werden von der Geschäftsstelle noch zugesandt.

\section{Workshop des Arbeitskreises Erlangen:}

7.-8. 11.98

Thema: Umgang mit posttraumatischen Belastungsstörungen Frau Dipl.-Psych. Miryam v. Samson-Himmelstjerna, 80538 München, Widenmayrstr. 6, Tel. 089 / 2913692

Als neue Lehrtherapeutin und neuen Lehrtherapeuten begrüßen wir ganz herzlich:

Anne Knappe, Dipl.-Psych., Jahrgang 1963, arbeitete 1990-1996 als wissenschaftliche Assistentin an der Universität Bamberg am Lehrstuhl Psychologie I bei Herrn Prof. Dr. Selg. Forschungs- und Arbeitsschwerpunkte waren hierbei die Prävention sexuellen Mißbrauchs (verschiedene Forschungsprojekte), in der Lehre entwicklungspsychologische und verhaltensmodifikatorische Themen. Freiberuflich seit 1991 in psychotherapeutischer Praxis in Lichtenfels/Ofr. Schwerpunkt der Praxistätigkeit ist die Arbeit mit Kindern, Jugendlichen und Frauen, insbesondere die Therapie mit sexuell traumatisierten Patientinnen. Darüber hinaus Therapie von Eßstörungen, Depressionen, Phobien und Persönlichkeitsstörungen. Tätig als Supervisorin in verschiedenen Institutionen des sozialen Bereichs seit 1993. Sachverständige für Glaubwürdigkeitsgutachten in Strafprozessen seit 1994. Seit 1987 (mit Pause) Mitglied der AVM, abgeschlossene VT-Weiterbildung bei der DGVT, klin. Psychologin/Psychotherapeutin und Supervisorin BDP, gesprächspsychotherapeutische Grundausbildung (GwG) und abgeschlossene Weiterbildung in personenzentrierter psychotherapeutischer und pädagogischer Arbeit mit Kindern und Jugendlichen (GwG).

Verzeichnis der Publikationen:

Brosowsky M, Hörsch U, Knappe A, Selg H: Trainingsprogramm für Erzieherinnen als Multiplikatoren von elternzentrierter Prävention sexuellen Mißbrauchs. Forschungsbericht, Bamberg, 1995.

Knappe A, Selg H: Prävention von sexuellem Mißbrauch an Mädchen und Jungen. Forschungsbericht, Bamberg, 1993.

Knappe A: Mit Kindern über sexuelle Mißhandlung reden, in Johns I (Hrsg): Zeit alleine heilt nicht. Freiburg, Herder, 1993.
Knappe A: Prävention von sexuellem Mißbrauch: Möglichkeiten und Grenzen in der Arbeit mit Eltern und Kindern, in Schubbe O (Hrsg): Therapeutische Hilfen gegen sexuellen Mißbrauch an Kindern. Göttingen, Vandenhoek \& Ruprecht, 1994.

Knappe A: Was wissen Eltern über Prävention sexuellen Mißbrauchs? in Marquard-Mau B (Hrsg): Schulische Prävention gegen sexuelle Kindesmißhandlung. Weinheim, Juventa, 1995.

Rainer Knappe, Dipl.-Psych., Jahrgang 1961, arbeitet seit 7 Jahren in freier Praxis in Lichtenfels/Ofr. im Bereich Therapie, Supvervision und Organisationsberatung. Mitbegründer eines beruflichen Aus- und Fortbildungsinstituts im gewerblichen Sektor. Mitglied der AVM seit 1987 in verschiedenen Vereinsfunktionen (AK-Leitung, AWANK, Vorstand der AVM-D), mit abgeschlossener VT-Weiterbildung bei der DGVT, klin. Psychologe/Psychotherapeut und Supervisor BDP, gesprächspsychotherapeutische Grundausbildung nach den Richtlinien der GwG. Arbeitsschwerpunkte im klinischen Bereich sind phobische, depressive und somatoforme Störungen, darüber hinaus die Arbeit mit Sexualstraftätern. Seit 1994 Lehrauftrag für Psychologie an der FH Bamberg im Fachbereich Sozialwesen in den Schwerpunkten Erziehungsund Familienhilfen.

Die Mitgliederversammlung der AVM-D am 28. 3. 1998 war gut besucht. Mit herzlichem Dank für seine langjährige Arbeit wurde der bisherige Kassierer, Herr Dipl.-Psych. Stefan Weinzierl, verabschiedet.

Dieses Amt hat ab sofort Frau Dipl.-Psych. Petra Gruhl übernommen, die wir hiermit willkommen heißen.

\section{Tagesordnung}

1. Begrüßung

2. Wahl der Versammlungsleitung

3. Bericht des Vorstandes

4. Bericht des Kassierers

5. Bericht der Kassenprüfer und Genehmigung des Kassenberichtes

6. Entlastung des Vorstandes

7. Bericht über den Stand der Akkreditierung

8. Bericht von Herrn Grimmel über Situation nach Ablauf der Übergangsregelung (altes Modell)

9. Abfrage des Finanz-Status der Arbeitskreise

10. Neuwahlen

a) des Kassierers

b) der Mitglieder der AWANK (mind. 3 Mitglieder). Beachte: Antrag zur Satzungsänderung § 9 (2) III

\section{Anträge}

- Satzungsänderung § 9 (2): Die Aus-, Weiterbildungs- und Anerkennungskommission (AWANK) besteht aus mindestens drei Personen. Zwei Mitglieder müssen als Lehrtherapeuten durch die AVM anerkannt sein oder eine ab- 
geschlossene Ausbildung in Verhaltenstherapie/Verhaltensmodifikation besitzen. Eines der Mitglieder kann auch von einem Arbeitskreisleiter besetzt werden. Die Mehrheit der AWANK-Mitglieder müssen Lehrtherapeuten sein. Der Kommission wird ein Mitglied des Vorstandes beratend zugeordnet

- Satzungsänderung $§ 8$ (5): . . . der Ausbildungs- und Anerkennungsrichtlinien als Verhaltenstherapeut/AVM ... löschen

- Aufhebung des festen Stundensatzes von Lehrtherapeuten (Antrag von Herrn Dr. Rudi Wagner)

- Jeder Ausbildungskandidat muß im 2. Ausbildungsabschnitt mindestens ein Plenum mitgestalten

- Erhöhung der Mitglieds- und Semesterbeiträge ab 1999

- Einführung des Einzugsverfahrens für Mitgliedsbeiträge ab 1999 für alle Mitglieder

12. Verschiedenes

Wie bereits auf der letzten Mitgliederversammlung am 28 . März '98 in Bamberg angekündigt wurde, will der Vorstand der AVM-D mit und für die Mitglieder der AVM einen vierteljährlichen Rundbrief auf die Beine stellen. Dieser Rundbrief soll als aktuelles Forum Informationen aus Vorstand, Arbeitskreisen und AWANK, Workshopankündigungen und Veranstaltungen, Kontaktadressen, eine Jobbörse und vieles andere mehr beinhalten. Jeder und jede, der/die Beiträge jeglicher Art zum AVM-Rundbrief beisteuern will, möchte sich bitte mit der Geschäftsstelle oder mit Herrn DP Marc Worbach - Friedensstraße 26, 97072 Würzburg, Tel. (09 31) 782694 - in Verbindung setzen. Der erste Rundbrief soll um den 1. August '98 in den einzelnen AKn verteilt werden; Redaktionsschluß ist der 20. Juli '98.

\section{Workshops - AVM AK Würzburg}

Psychosexualtherapie in der allgemeinen verhaltenstherapeutischen Praxis

mit Frau Dr. Monika Vogelsang, Psychosomatische Fachklinik Münchwies

Inhalt:

Ziel des Workshops ist es, die Vermeidung sexueller Themen in der verhaltenstherapeutischen Anwendung der WorkshopteilnehmerInnen zu reduzieren und hier eine offene und empathische Integration der Sexualität der PatientInnen zu ermöglichen. Spezielles Wissen soll vermittelt und eine Reduktion sexualbezogener Ängste soll bewirkt werden.

Besondere Beachtung findet die Ätiologie und Therapie der sexuellen Funktionsstörungen sowie der Paraphilien. Schließlich wird auf ethische Fragen eingegangen.

Zeiten: Sa.: 31. 10. 98, 11.00-13.00 Uhr, 14.00-19.30 Uhr So.: 01. 11. 98, 9.00-12.00 Uhr, 13.00-15. $30 \mathrm{Uhr}$ jeweils 1 Stunde Mittagspause, ${ }^{1} / 2$ Stunde Kaffeepause

Ort: $\quad$ Praxis Purucker, Huttenstr. 10, 97072 Würzburg
AVM-Geschäftsstelle:

Lehrstuhl für Klinische

Psychologie der Universität

Markusplatz 3

D-96045 Bamberg

\section{Deutsche Gesellschaft für Verhaltensmedizin (DGVM)}

Der 7. Kongreß der Deutschen Gesellschaft für Verhaltensmedizin und Verhaltensmodifikation findet unter dem Thema Verhaltensmedizin an der Schwelle zum neuen Jahrtausend Entwicklungen und Perspektiven vom 10. bis 13. März 1999 in Prien am Chiemsee statt.

Nach mehreren universitären Tagungsorten wird der Kongreß dieses Mal in einem mehr praxisorientierten Ambiente stattfinden. Gastgeber ist die Medizinisch-Psychosomatische Klinik Roseneck, die seit vielen Jahren Konzepte der Verhaltensmedizin umsetzt und diese auch wissenschaftlich evaluiert.

Der Kongreß soll allen Teilnehmern eine stimulierende Auseinandersetzung mit brandaktuellen Entwicklungen der Verhaltensmedizin ermöglichen. Neueste Ergebnisse aus wichtigen Forschungsarbeiten werden präsentiert und moderne Behandlungsansätze dargestellt. Gemäß des interdisziplinären Ansatzes der Verhaltensmedizin richtet sich der Kongreß gleichermaßen an Psychologen und Ärzte verschiedener Fachrichtungen. Der Kongreß wird eine Mischung aus Hauptvorträgen, Symposien und Postersessions bieten.

Folgende internationale Referenten konnten für Hauptvorträge gewonnen werden:

- Prof. Pavel Jastreboff (Baltimore, USA): Tinnitus

- Prof. David Clark (Oxford, UK): Verhaltenstherapie

- Prof. Per Björntorp (Göteborg, Schweden): Adipositas

- Prof. Simon Wessely (London, UK): Chronic Fatigue Syndrome

- Prof. Douglas Drossman (Chappel Hill, USA): Reizdarmsyndrom

- Prof. Dennis Turk (Seattle, USA): Schmerz

Symposien sind zu folgenden Themen geplant:

- Eßstörungen und Adipositas

- Gynäkologie und Fertilitätsmedizin

- Somatoforme Störungen

- Tinnitus

- Schmerz

- Gastrointestinale Erkrankungen

- Hauterkrankungen

- Kardiovaskuläre Erkrankungen

- Schlafstörungen

- Psychotherapie und Arbeitswelt

- Therapieforschung in der Verhaltensmedizin 
- Psychologische und psychobiologische Grundlagen des Lernens

- Epidemiologie in der Verhaltensmedizin

- Verhaltensmedizin bei Kindern und Jugendlichen

- Verhaltensmedizin bei alten Menschen

Bitte reichen Sie alle Anmeldungen für Beiträge auf MSDOS-formatierten oder Macintosh-kompatiblen Disketten ein und verwenden Sie eine Standardsoftware für Textverarbeitung (z. B. Word). Die Anmeldung für einen wissenschaftlichen Beitrag muß nach folgendem Muster aufgebaut sein: DE: Vortrag oder Poster

TI: Neue Ansätze der Schmerztherapie

AU: Karl Kern und Herbert Winter

IN: Institut für Medizinische Psychologie, Abteilung, Straße, Postleitzahl, Ort
AB: Abstract von maximal 250 Wörtern mit folgendem Aufbau: Fragestellung, Methoden, Ergebnisse, Diskussion, maximal 2 Literaturangaben

Thematisch zusammenhängende Beiträge können auch als Symposien angemeldet werden.

Symposien können maximal 8 Einzelbeiträge umfassen.

Weitere Informationen:

Sekretariat Prof. Dr. M. Fichter

Klinik Roseneck

Am Roseneck 6

83209 Prien

Tel.: 08051 / 68-35 10, Fax: 08051 / 68-35 32

eMail: 080516010-0001@T-online.de 